\title{
NASCA Microscopy: Super-Resolution Mapping of Chemical Reaction Centers
}

\author{
Gert De Cremer, Bert F. Sels, Dirk E. De Vos, Johan Hofkens, \\ and Maarten B.J. Roeffaers
}

\begin{abstract}
Recently fluorescence microscopy has been introduced in the field of catalysis to study their dynamic molecular processes under in situ conditions with high spatial and temporal resolution. Because of the unique sensitivity down to the single molecule level, fluorescence microscopy allows to observe and localize chemical transformations with a subdiffraction-limited resolution. This chapter describes the use of fluorogenic probe molecules to visualize single chemical conversions using fluorescence microscopy. Special attention is paid to how visualization of single chemical conversions can yield super-resolution images beyond the diffraction limit.
\end{abstract}

Keywords Fluorogenic substrate - Heterogeneous catalysis · Single catalytic turnover counting - Wide-field fluorescence microscopy

G. De Cremer, B.F. Sels, and D.E. De Vos

Center for Surface Chemistry and Catalysis, Katholieke Universiteit Leuven, 3001 Heverlee, Belgium

J. Hofkens

Laboratory for Photochemistry and Spectroscopy, Katholieke Universiteit Leuven, 3001

Heverlee, Belgium

M.B.J. Roeffaers $(\bowtie)$

Center for Surface Chemistry and Catalysis, Katholieke Universiteit Leuven, 3001 Heverlee, Belgium

Laboratory for Photochemistry and Spectroscopy, Katholieke Universiteit Leuven, 3001

Heverlee, Belgium

e-mail: maarten.roeffaers@biw.kuleuven.be 


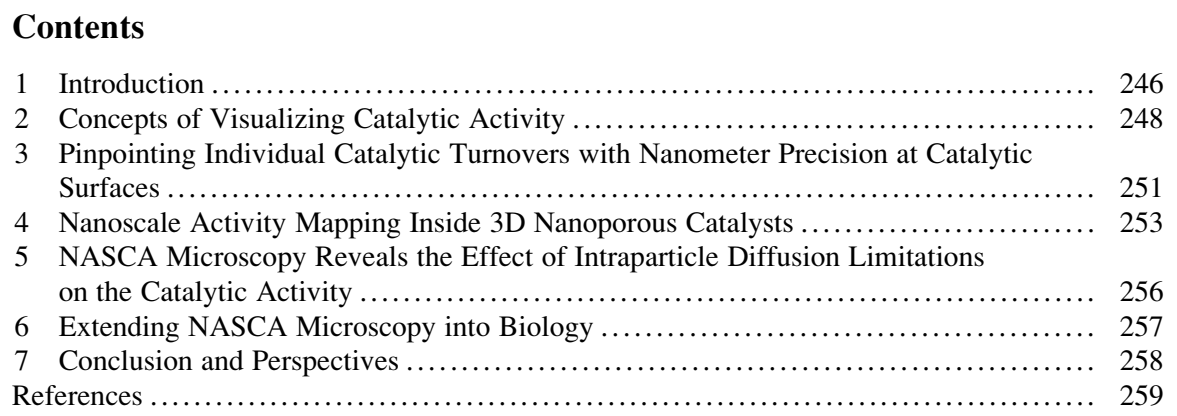

\section{Abbreviations}

$\begin{array}{ll}\text { BODIPY } & \text { 4,4-Difluoro-4-bora-3a,4a-diaza-s-indacene } \\ \text { GSDIM } & \text { Ground-state depletion and single molecule return } \\ \text { LDH } & \text { Layered double hydroxide } \\ \text { MRI } & \text { Magnetic resonance imaging } \\ \text { NASCA } & \text { Nanometer accuracy by stochastic chemical reactions } \\ \text { PALM } & \text { Photo-activated localization microscopy } \\ \text { PET } & \text { Photo-induced electron transfer } \\ \text { PSF } & \text { Point-spread-function } \\ \text { STED } & \text { Stimulated emission depletion microscopy } \\ \text { STORM } & \text { Stochastic optical reconstruction microscopy } \\ \text { TIRF } & \text { Total internal reflection fluorescence }\end{array}$

\section{Introduction}

The performance of a catalytic particle is determined by its chemical composition, the structure at the atomic scale, and the accessibility [1]. In order to better predict and understand the influence of these parameters on the catalytic performance, a broad range of physicochemical characterization techniques has been developed $[1,2]$. In general, such in-depth characterizations focus on determining the catalysts' structure and chemical composition, preferentially at the atomic scale and under catalytically relevant conditions. For instance, detailed structural characterization of catalyst particles is routinely accessible via electron microscopy. Furthermore, it has recently become possible to obtain compositional and structural information on the catalyst particle using scanning probe [3] and X-ray microscopy [4].

In stark contrast to these detailed structural and chemical pictures of (inorganic) catalytic surfaces, insight into the organic chemical transformations at these surfaces is mostly limited to bulk analysis of reaction mixtures by, for example, chromatographic or mass spectrometric analysis. At best these conversions can be 
observed with sub-millimeter spatial resolution using vibrational spectroscopy or MRI [5, 6]. This discrepancy in resolution and sensitivity hampers a one-to-one linking of catalytic activity to local catalyst properties.

To overcome this lacuna, microscopic techniques that combine an excellent spatial and temporal resolution with an exceptional sensitivity for organic compounds are necessary [7-9]. Similar requirements apply when studying biomedical samples in detail, and for such studies the use of fluorescence microscopy has proven to be a major advance. The field of fluorescence microscopy has witnessed strong technical and practical developments over the past decade, many of these advances aiming for improved sensitivity and enhanced spatial resolution. As a result, single molecule sensitivity has become widely available, and various diffraction-unlimited or super-resolution imaging schemes have been reported [10,11]. While biomedical super-resolution imaging schemes such as PALM, STORM, and STED rely on the photostimulated switching of molecules between an emissive and a non-emissive state, diffraction-unlimited imaging of heterogeneous catalysts in NASCA microscopy, or Nanometer Accuracy by Stochastic Chemical reActions microscopy, uses the isolated signals of individual converted fluorogenic probe molecules [9]. In this approach, visualization and accurate localization of individual catalytic turnovers, occurring stochastically,
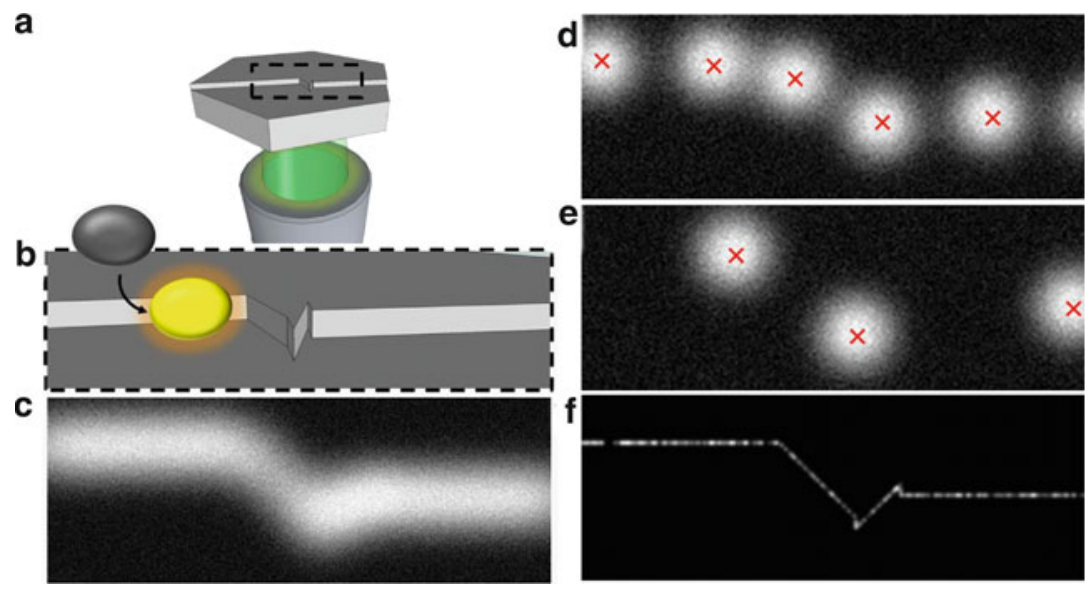

Fig. 1 Nanometer Accuracy by Stochastic Chemical reActions (NASCA) microscopy. (a) Catalytic materials contain a wide range of different facets and defects on which chemical reactions take place. (b) Fluorogenic substrates allow visualizing chemical conversions down to the single molecule level. (c) The resolution of traditional optical microscopy is limited to a few hundred nanometers. As a consequence, chemical events occurring on nanometer-sized features like this crystal edge are projected as broad zones of activity. (d, e) NASCA microscopy relies on recording the stochastic catalytic conversions of fluorogenic substrates at the single turnover level in real time. The exact location of each of these individual chemical reactions can be approximated with nanometer precision (red crosses) by mathematical fitting of the diffraction-limited pointspread-function (PSF). (f) Accumulation of these approximated reaction positions over consecutive frames allows reconstructing a high resolution optical image 
allow reconstructing high resolution images of the active domains of the material under study $[12,13]$.

This chapter highlights this novel high resolution optical microscopy method based on localization of individual catalytic conversions of fluorogenic and chromogenic substrates. Next to reconstructing super-resolution images, elegant applications are illustrated such as quantification of the catalytic activity with preservation of spatial information at the nanometer scale. NASCA microscopy is technically simple since only one excitation wavelength is required avoiding the need for spatiotemporal overlap between different light sources, as is required in most other super-resolution fluorescence microscopy approaches. Figure 1 graphically explains the concept of NASCA microscopy. In this figure, it is obvious that accumulation of the approximated position of all the visualized individual chemical reactions allows reconstruction of a much more detailed image (Fig. 1f) than the traditional diffraction-limited one (Fig. 1c).

\section{Concepts of Visualizing Catalytic Activity}

The chemical conversion of nonfluorescent probes into strongly fluorescent reaction products is an ideal strategy to visualize and locate catalytic conversions inside (porous) materials. Such substrates are called fluorogenic and chromogenic compounds. Chromogenic substrates are noncolored species and only after their conversion they are able to absorb visible light. A fluorogenic reagent is a nonfluorescent chemical compound that can be chemically converted into a strongly fluorescent product molecule. Such compounds have been widely used to assess enzymatic activity [14]. Recently a few of such reagents have found their application in the characterization of catalyst particles using fluorescence microscopy $[9,15,16]$. In this context, the names fluorogenic and chromogenic are defined in a very broad sense: they also encompass molecules which as a result of a chemical transformation show a dramatic shift in their absorption spectrum and/or a drastic change in fluorescence quantum yield. This shift in photophysical properties results in a clear switch between a non-emissive and an emissive state at a selected excitation and detection wavelength. Simplified the molecule is converted from a non-detectable to a detectable state. Under the right conditions, the latter state can be visualized preferentially with single molecule sensitivity.

The first fluorogenic reagent described in literature to visualize individual chemical turnovers at a solid catalyst particle is the nonfluorescent acetic ester of fluorescein. Cleaving off the acetate groups, for example, according to basecatalyzed hydrolysis and transesterification reactions, yields the brightly emissive fluorescein (Fig. 2a), which can easily be detected at the single molecule level with current top-end fluorescence microscopes [17]. Fluorescein esters were already well known from biomedical research where they are used, for example, as cell viability probe reporting on the activity of intracellular esterases. Next to fluorogenic esters of fluorescein, there are many derivatives like ethers, amides, etc. of xanthene 
a

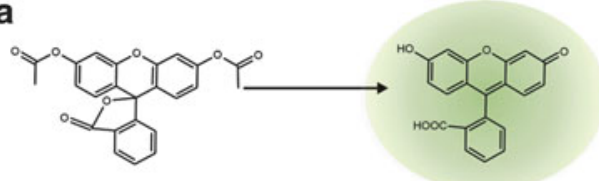

b
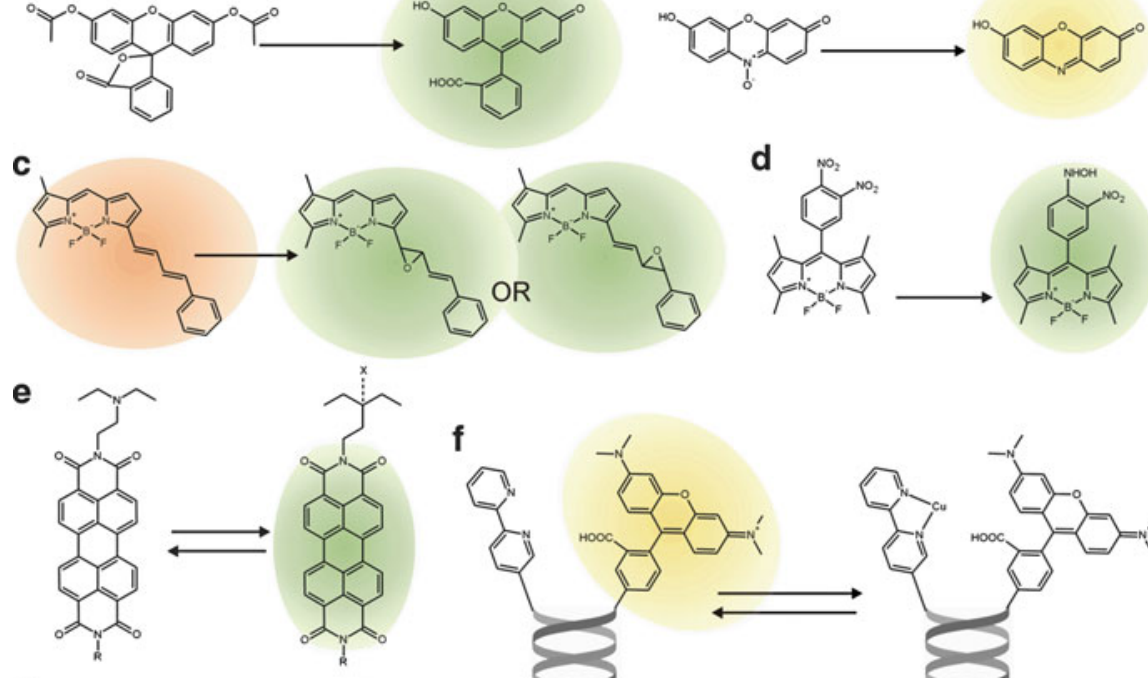

g
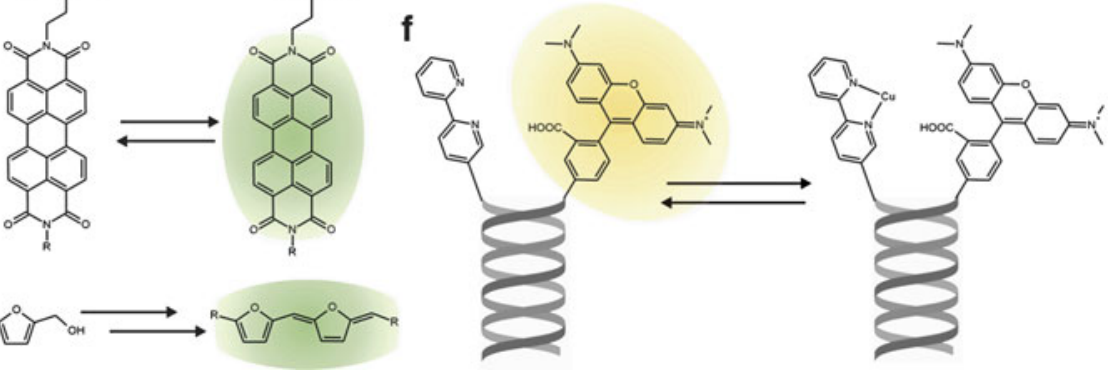

Fig. 2 Examples of fluorogenic probe reactions for NASCA microscopy [9, 12, 13]. (a) Formation of fluorescein from the fluorogenic substrate fluorescein diacetate [17]. (b) Reduction of the fluorogenic resazurin to the yellow fluorescent resorufin [18]. (c) Conversion of the red fluorescent phenylbutadiene-BODIPY to the green fluorescent epoxidized product $[12,13]$. (d) Photocatalytic reduction of the fluorogenic dinitrophenyl-BODIPY yields a green fluorescent product [19]. (e) An amine in the unbound state quenches the fluorescence of a perylene diimide (PDI) chromophore via photo-induced electron transfer (PET). This process is inhibited when the free electron pair of the amine binds a proton or a metal ion, which is indicated by $X$, thereby restoring the fluorescence of PDI [20]. (f) Fluorescence quenching by $\mathrm{Cu}$ (II) complexation on a nearby bipyridine group [21-23]. (g) Catalytic oligomerization of furfuryl alcohol results in strongly fluorescent reaction products $[13,24,25]$

dyes known. This wide variety of fluorogenic substrates is further extended with nonfluorescent oxidized or reduced versions of chromophores. Well-known examples of such chromophores are rhodamine and its reduced version dihydrorhodamine and resorufin and the oxidized form resazurin (Fig. 2b) [18], etc. The commercial availability of many of these dyes enables to target acid and base as well as redox-type catalytic reactions.

Recent reports have also shown that an intelligent derivatization and functionalization of well-known chromophores can also be used to generate reaction-specific fluorogenic reagents. De Cremer et al. reported on monitoring individual epoxidation reactions using a phenylbutadiene-functionalized BODIPY dye (Fig. 2c) $[12,26]$. The addition of this phenylbutadiene-conjugated system to the central chromophore induces a red shift of about $80 \mathrm{~nm}$ in both the absorption and fluorescence spectrum compared to the original dye [27]. Selective epoxidation of 
the butadiene bridge of this substituted dye shifts the absorption and fluorescence maximum back toward the original chromophore. Excitation near the absorption maximum of these products and fluorescence detection around its emission maximum allows the detection of individual reaction products even in the presence of a large excess of phenylbutadiene-functionalized BODIPY reagents in the surrounding solution.

Majima and coworkers also used BODIPY dye as a starting point to generate a reduction sensitive probe molecule (Fig. 2d) [19]. Addition of a 3,4-dinitrophenyl group quenches the fluorescence of the chromophore by photo-induced electron transfer (PET) from the excited chromophore to the dinitrophenyl moiety. Reduction of one nitro group to a hydroxylamine makes this intramolecular electron transfer unfavorable, hence restoring the fluorescence of the BODIPY chromophore. This makes the 3,4-dinitrophenyl functionalized BODIPY an excellent fluorogenic substrate to study the exact location of, for example, photocatalytic reductions.

Electron or energy transfer processes can also be used to monitor reversible binding and unbinding reactions with single molecule sensitivity. One such example is the protonation-deprotonation of amines (Fig. 2e) [20]. While amines with a free electron pair can quench the fluorescence of an excited chromophore in the vicinity via a PET process, protonation of the amine hinders this process, hence restoring the fluorescence intensity. Ions like $\mathrm{Cu}$ (II) can also quench the fluorescence of nearby chromophores. Functionalization of a strong chromophore with a copper-binding group like a bipyridine ligand results in a reversible fluorescent switch in which the fluorescence emission is determined by the binding or unbinding of $\mathrm{Cu}$ (II) ions (Fig. 2f) [23]. Thus a wide variety of fluorogenic chromophores can be thought of and designed starting from existing chromophores.

Next to these fluorogenic derivatives of well-known chromophores, a series of probe reactions has been described based on small organic molecules, like furfuryl alcohol [8, 13, 24, 28], styrenes [29, 30], thiophenes [31], etc., which via catalytic action are converted into extended conjugated systems (Fig. 2g). These oligomers are very efficient to probe, for example, acid-catalyzed conversions in the nanopores of zeolites. Stabilization of the oligomer by tight confinement in the pores enhances the fluorescence emission. Even though these reactions have shown their power as catalytic activity reporters at the single catalytic particle level, almost all studies average out the fluorescence intensity over a multitude of product molecules located within a diffraction-limited area. So far only one of these reactions was successfully monitored at the single molecule level [13]. The acidcatalyzed furfuryl alcohol condensation yields fluorescent oligomers which are sufficiently bright to detect individual reaction products.

In conclusion, the use of fluorogenic substrates makes it possible to visualize individual catalytic turnovers and approximate the exact position of this conversion with nanometer precision (see Fig. 1). Tuning the substrate concentration and balancing its conversion rate with the speed of disappearance of the fluorescent products due to photobleaching or diffusion allow mapping out and even quantifying local reaction rates at the single conversion level. 
When no suitable chromogenic reactant can be found, one can still rely on the use of a spectator fluorophore bound to the reagent. This is for instance demonstrated by the group of Blum for a BODIPY-bound platinum complex [32-35]. By imaging the fluorescence in total internal reflection mode, TIRF microscopy, only species physiand/or chemisorbed in close distance (few tens of nanometers) to the cover glass-liquid interface can be detected. Although this approach is thus not suitable to monitor reactions deep inside catalytic materials, it provides a useful alternative to the use of chromogenic substances when interested in surface processes.

\section{Pinpointing Individual Catalytic Turnovers with Nanometer Precision at Catalytic Surfaces}

The influence of structural features of solid catalyst particles on their local catalytic activity was for the first time directly observed on layered double hydroxide (LDH) crystals [17]. The LDH crystals used in this study consist of sheets of aluminum hydroxides in which aluminum(III) occupies two of three octahedrons. Introduction of lithium in the residual octahedrons results in positively charged layers which gives the material an interesting anion exchange capacity. Presence of hydroxide ions in the galleries between the sheets gives solid base catalyst functionality to the structure, illustrating its catalytic potential [36]. Clearly the crystals have different crystal facets, being the (large) basal plane and the lateral faces, with their own specific environments and a varying accessibility which will unavoidably lead to differences in local catalytic activity.

The base-catalyzed conversion of the fluorogenic fluorescein ester (Fig. 2a) was carried out in two different solvents that also act as reagent for the conversion into the strongly emissive fluorescein. When operated in an aqueous environment, the fluorogenic ester is hydrolyzed into fluorescein and free acetate, whereas in an $n$-butanol the same fluorescein is formed together with the corresponding acetic ester in a transesterification reaction. The strongly fluorescent reaction product allows the observation of both reactions with single turnover sensitivity. Interestingly, for the hydrolysis reaction catalytic turnovers were mainly observed at the crystal's lateral faces, whereas for the transesterification in $n$-butanol the whole outer surface participated in the catalytic conversion. The reconstructed NASCA images clearly show this difference between both reactions (Fig. 3).

Next to reconstructing such activity maps, NASCA microscopy (see also Fig. 1) also allows the quantification of the local catalytic rates and this by simple counting of the amount of turnovers for a selected area during a certain time interval. The transesterification reaction showed a homogeneously distributed activity over the whole outer surface (Fig. 3a-c). This catalytic activity increases linearly with an increased substrate concentration. On the other hand, the hydrolysis activity was for $85 \%$ confined to the lateral crystal faces (Fig. 3d-f). During these experiments, the diffusion of the fluorescein product over the catalyst surface could also be observed. 

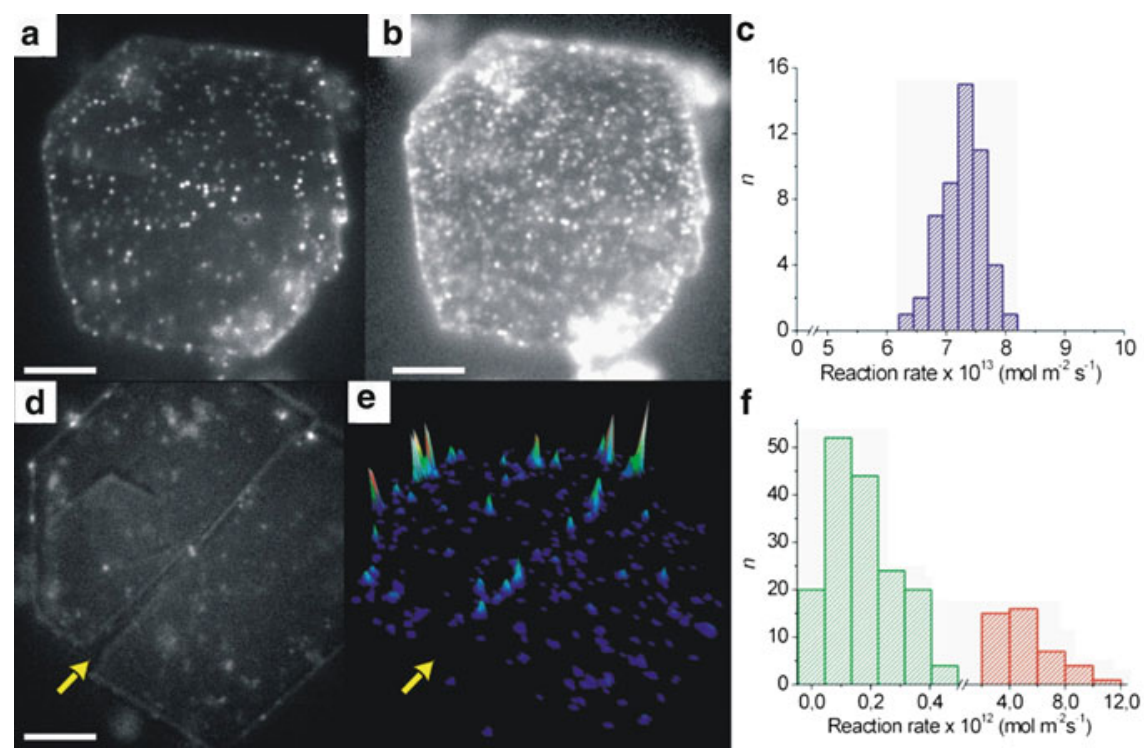

Fig. 3 (a, b) Fluorescence image during the transesterification of the fluorogenic fluorescein diacetate ester with $n$-butanol by a $\mathrm{LDH}, 40 \mathrm{nM}$ (a) and $700 \mathrm{nM}$ (b) substrate concentration. The bright spots correspond to single catalytic reaction products at the LDH outer surface. (c) Quantification of the initial transesterification rates for $1 \mu \mathrm{m}^{2}$ domains. (d) Fluorescence image of a LDH crystal during hydrolysis of $600 \mathrm{nM}$ C-FDA. (e) Accumulated intensity on the same crystal over 256 consecutive images, highlighting the higher contribution of the lateral faces to the total hydrolysis activity. (f) Quantification of the initial hydrolysis rates for $1 \mu \mathrm{m}^{2}$ domains. The fast subpopulation ( $r e d$ ) corresponds to active domains located at lateral faces, whereas the basal planes are responsible for the slow population (green). Scaling bars: $5 \mu \mathrm{m}$. Reproduced with permission from [17]. Copyright 2006, Nature Publishing Group

A large fraction of the molecules $(90 \%)$ showed a high surface mobility $(D=3 \times$ $10^{-14} \mathrm{~m}^{2} \mathrm{~s}^{-1}$ ), whereas for $10 \%$ of the molecules the observed displacements are smaller than the experimental error.

A second example where NASCA microscopy was exploited is in the field of electrocatalysis [18]. Here, single electrocatalytic reductions by single-walled carbon nanotubes (SWNT) were observed with a wide-field fluorescence microscope using the conversion of the fluorogenic resazurin into the strongly fluorescent resorufin (see Fig. 2b). By localizing and counting these conversions on the long nanotubes (length of a few micrometer), NASCA microscopy revealed that the reactions are concentrated in areas of about $20 \mathrm{~nm}$ diameter, which equals the accuracy limit of this study. From this result it was thus concluded that the electrocatalytic reductions occur at discrete reaction sites with a nanometer-size dimension that are present along the SWNTs. Moreover, careful analysis of the kinetics of the reaction process revealed a large heterogeneity in reactivity among these reactive sites reflecting the heterogeneity in electronic properties. 

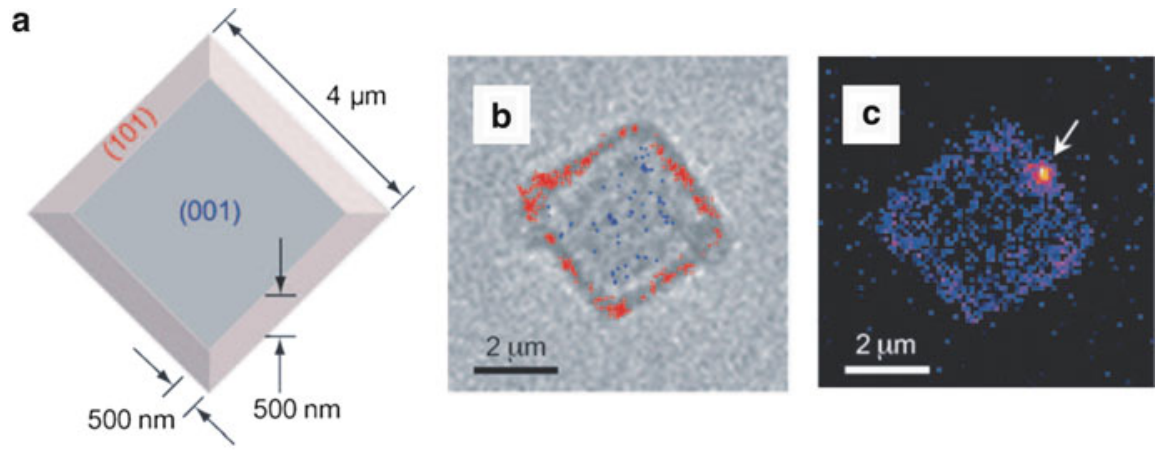

Fig. 4 (a) Schematic of a single micrometer-sized anatase $\mathrm{TiO}_{2}$ crystal with preferential (001) facets. (b) Optical transmission image of a single $\mathrm{TiO}_{2}$ crystal, and (c) fluorescence image of the same crystal in an argon saturated $2.0 \mathrm{mM}$ dinitrophenyl-BODIPY solution under $488 \mathrm{~nm}$ laser and UV irradiation. The red and blue dots in image (b) indicate photocatalytic turnovers located on the (101) and (001) surfaces, respectively, observed during $3 \mathrm{~min}$ of irradiation. The fluorescent products were localized by mathematical fitting of the PSF. The arrow in (c) indicates an individual reaction product. Copyright Wiley-VCH Verlag GmbH \& Co. KGaA. Reproduced with permission from [19]

Majima and coworkers also used this single catalytic turnover detection scheme to precisely map redox reactions at the outer surface of individual photocatalyst particles $[19,37,38]$. As fluorogenic probe molecule they used the 3,4-dinitrophenylsubstituted BODIPY dye, where the fluorescence of the excited chromophore is quenched by an intramolecular PET to the 3,4-dinitrophenyl moiety (Fig. 2d). Photocatalytic reduction of one of the nitro's to a hydroxylamine restores the chromophore's emissive properties (Fig. 2d). These photocatalytic events can be selectively visualized on individual micrometer-sized anatase particles (Fig. 4) [19]. By pinpointing the different turnovers on top of the optical transmission image, it becomes clear that the majority of the photocatalytic conversions takes place on the (101) plane. Quantification of these conversions learns that the photocatalytic reduction of this probe at the (101) plane is about three times more efficient than at the (001) plane (Fig. 4b). This observation is linked to the spatially selective migration of photogenerated electrons and holes.

\section{Nanoscale Activity Mapping Inside 3D Nanoporous Catalysts}

The previous examples nicely demonstrate the strength of NASCA microscopy for observing individual catalytic events and quantifying the surface activity. However, many heterogeneous catalysts have complex three-dimensional structures often with a complex intracrystalline nanoporous network to maximize the catalytically active surface. Because of size restrictions, these nanopores cannot accommodate the well-known polycyclic aromatic chromophores commonly used in single 
a
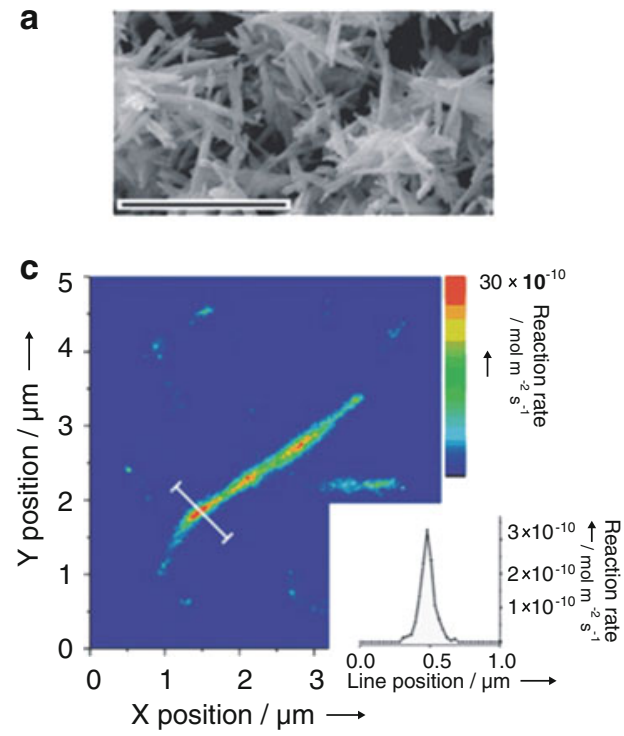

b

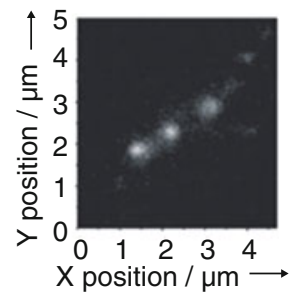

d

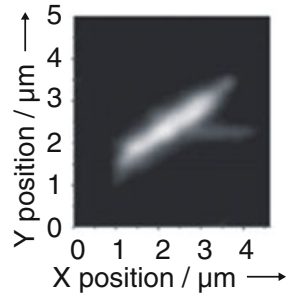

Fig. 5 (a) SEM image of needle-like ZSM-22 crystallites (average particle width: $120 \mathrm{~nm}$; scale bar: $5 \mu \mathrm{m}$ ). (b) Individual catalytic conversions of furfuryl alcohol on ZSM-22 crystallite. (c) Diffraction-limited image resulting from accumulation of all fluorescence observed during $1,000 \mathrm{~s}$ of reaction. (d) NASCA map showing catalytic reactivity for $20 \times 20 \mathrm{~nm}^{2}$ pixels. The intensity of the bins is determined by the amount of reactions localized in each bin using the high resolution scheme. Inset: local reaction rate as measured along the white line. A width of about $100 \mathrm{~nm}$ is obtained, corresponding to the real width of the imaged structure. Copyright Wiley$\mathrm{VCH}$ Verlag $\mathrm{GmbH} \& \mathrm{Co}$. KGaA. Reproduced with permission from [13]

molecule fluorescence studies. As a result, observation of single catalytic turnovers in these porous networks critically depends on the identification of small reagent molecules that are converted into sufficiently bright fluorescent product molecules that can be distinguished from the background noise. Surprisingly, furfuryl alcohol is such a reagent. Roeffaers et al. have shown that the pore-entrapped reaction products of an acid-catalyzed oligomerization reaction are sufficiently bright to enable single molecule observations in a wide-field fluorescence microscope [13].

First, the catalytic conversion of furfuryl alcohol inside the pores of needleshaped H-ZSM-22 crystallites was observed (Fig. 5). In every frame, bright emissive spots originating from individual oligomeric reaction products were observed (Fig. 5b). Using the single molecule localization techniques, these reaction sites were accumulated over 10,000 frames $(1,000 \mathrm{~s})$. The resulting NASCA microscopy image projects the catalyst particle with its real width of approximately $100 \mathrm{~nm}$ (Fig. 5c). In contrast, a normal diffraction-limited fluorescence image transforms these $100 \mathrm{~nm}$ thin catalytic rods as approximately $500 \mathrm{~nm}$ wide objects (Fig. 5d).

Furthermore, NASCA microscopy makes it possible to quantify the local catalytic activity at the nanometer scale. Such reactivity maps clearly uncover zones with different reactivity within an individual ZSM-22 needle. NASCA microscopy 

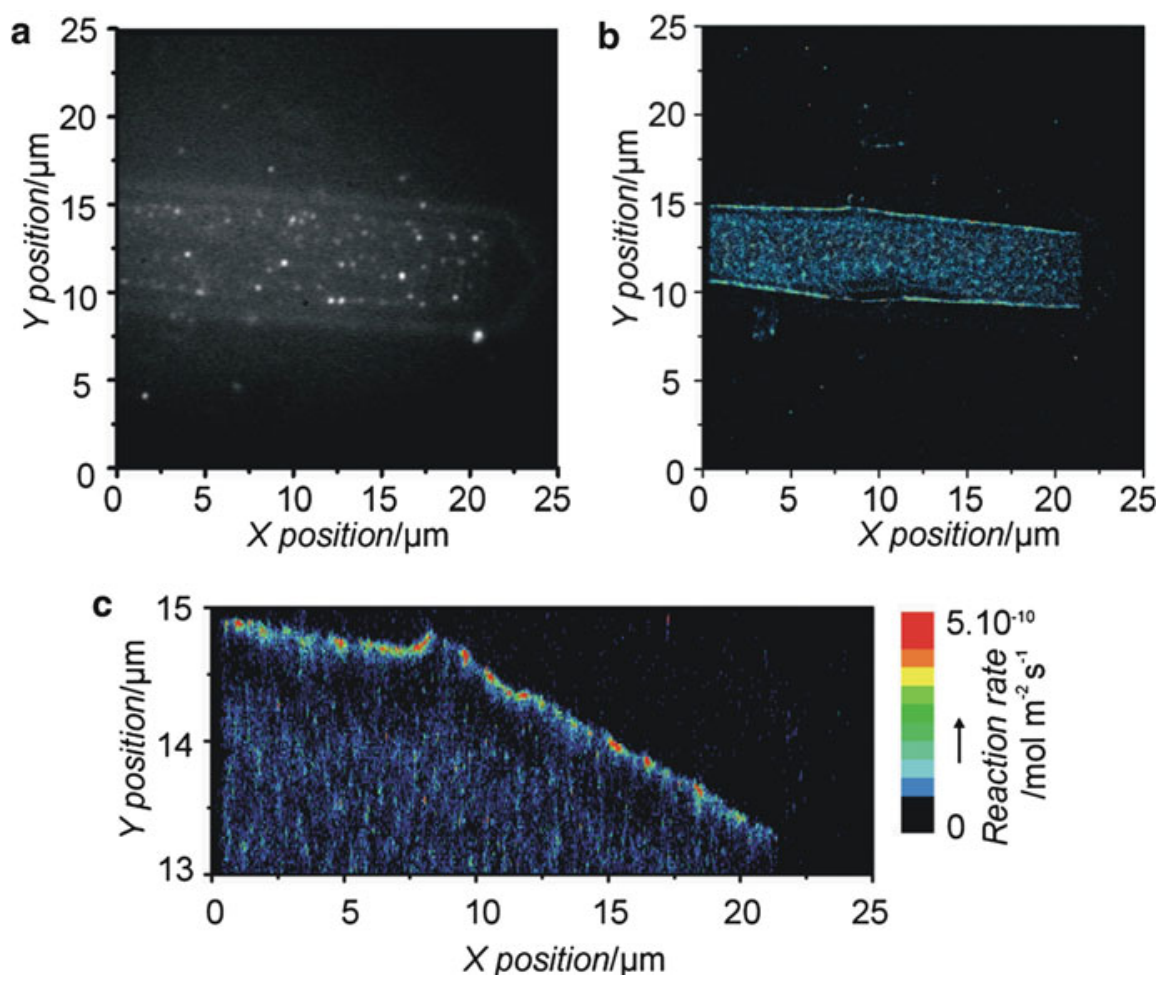

Fig. 6 (a) Individual fluorescent product molecules on a ZSM-5 crystal. The contours of the crystal are vaguely visible because of a small amount of unfiltered scattered light. (b) NASCA map showing catalytic reactivity for $20 \times 20 \mathrm{~nm}^{2}$ pixels. (c) Details of the edge of the crystals intergrowth where a nanometer-sized edge is present which shows a tenfold enhanced reactivity. Copyright Wiley-VCH Verlag GmbH \& Co. KGaA. Reproduced with permission from [13]

is the first technique that gives access to local, nanoscale catalytic reactivity inside porous structures, making it the true organic counterpart of high resolution inorganic imaging techniques like electron microscopy.

This same furfuryl alcohol oligomerization reaction was also used to visualize nanoscale reactivity profiles of the large hexagonal facets of ZSM-5 (Fig. 6). Such NASCA micrographs reveal a strongly enhanced catalytic reactivity in a central zone in the middle of these facets. Furthermore, zooming in on these super-resolution NASCA maps uncovers the presence of a very narrow zone with tenfold increased activity, lining the highly active central zone. The origin of this enhanced catalytic activity was revealed in a detailed electron microscopic study: the central zone with elevated activity overlapped with the $90^{\circ}$ intergrowths present in the main crystal body. These intergrowths are also outlined by distinct $50-100 \mathrm{~nm}$ high steps in which fresh furfuryl alcohol reagent can diffuse into the catalyst porous network from two perpendicular directions. This enhanced supply of reagents at the edges of the intergrowth may explain the local increase in turnover event frequency (Fig. 6). 
The pore-entrapped reaction products rapidly accumulate near the outer surface of the zeolite particles; this restricts current studies to the observation of catalytic reactivity to the initial stages of the process. Use of reactions in which the formed product can freely diffuse away would solve this problem, enabling observation of the catalytic activity during steady-state conditions as is shown in the following part.

\section{NASCA Microscopy Reveals the Effect of Intraparticle Diffusion Limitations on the Catalytic Activity}

The above-mentioned NASCA approach was recently applied to evaluate the catalytic performance of a Ti-MCM-41 epoxidation catalyst in the presence of tert-butyl hydroperoxide $[12,26]$. This catalyst consists of micrometer-sized particles with parallel mesoporous channels of about $2.5 \mathrm{~nm}$ in diameter. As a probe reaction, a phenylbutadienyl-substitued BODIPY dye (PBD-BODIPY) was used. This red fluorescent dye undergoes a strong blue-shift upon epoxidation (Fig. 2c). Thus, by spectral selection one can selectively monitor the formation of individual PBD-BODIPY molecules inside the catalytic particles. Figure 7 shows a representative picture of individual reaction positions, obtained by the NASCA approach, overlaid with a transmission image of the corresponding catalyst particle. Surprisingly, the NASCA image reveals that the reaction turnovers are almost exclusively confined to the outer few hundred nanometers of the catalyst particles. These results were interpreted in terms of diffusion limitations: the influx of PBDBODIPY molecules cannot keep up with the fast rate of conversion of these molecules. As a result, a steep concentration gradient throughout the particle is observed, even in steady-state conditions. In the case of a first-order reaction, the

a

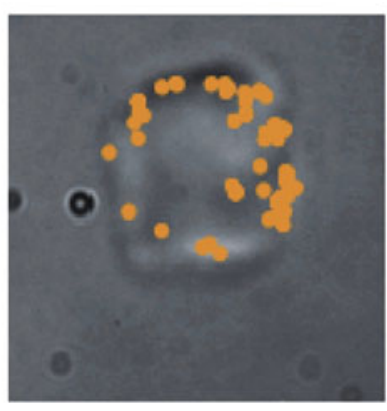

b

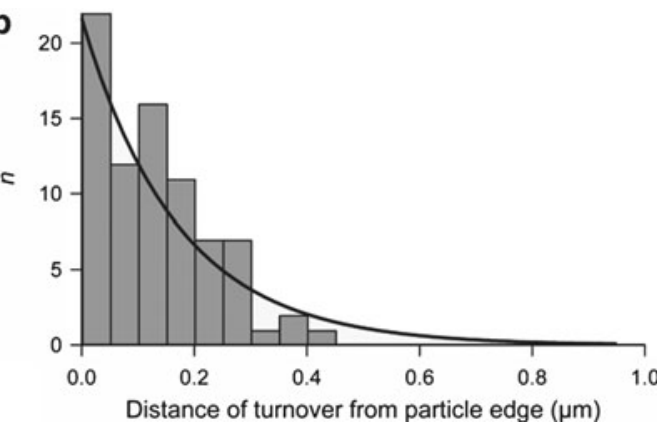

Fig. 7 (a) High-accuracy localized reaction positions inside an individual Ti-MCM-41 particle (particle dimension: $\pm 3 \mu \mathrm{m}$ ). (b) The histogram of the distances between the fitted position and the particle's edge directly reflects the reagent concentration profile inside the particles and can thus be directly related to the degree of diffusion limitation. Adapted with permission from $[12,26]$ 
spatial distribution of detected reaction events, that is, the NASCA map, directly reflects this concentration gradient (Fig. 7b) [12, 26]. Therefore, from the NASCA map, the degree of diffusion limitation can be directly quantified, as demonstrated by De Cremer et al. The catalyst was found to work at only $30-40 \%$ of its maximum capacity due to diffusion limitation. Moreover, as the single molecule approach for activity monitoring yields an absolute number of reaction events per unit of time, the reaction rate constant could be calculated, and together with the spatial distribution of the reactivity, an absolute value of the intraparticle diffusion coefficient could be obtained as well. Such values are extremely hard to obtain by the traditional "ensemble-averaged" experiments.

Thus, one NASCA microscopy experiment yields enough information to directly map and quantify the local reaction rates, and to determine effective diffusion coefficients, rate constants, and reactions efficiencies of individual catalyst particles even in steady-state conditions.

\section{Extending NASCA Microscopy into Biology}

Reaction cycles containing a fluorescent species can also be used to visualize and localize biological structures. Schwering et al. used the reversible $\mathrm{Cu}$ (II) complexation reaction (Fig. 2f) to switch the fluorescence of a nearby chromophore between
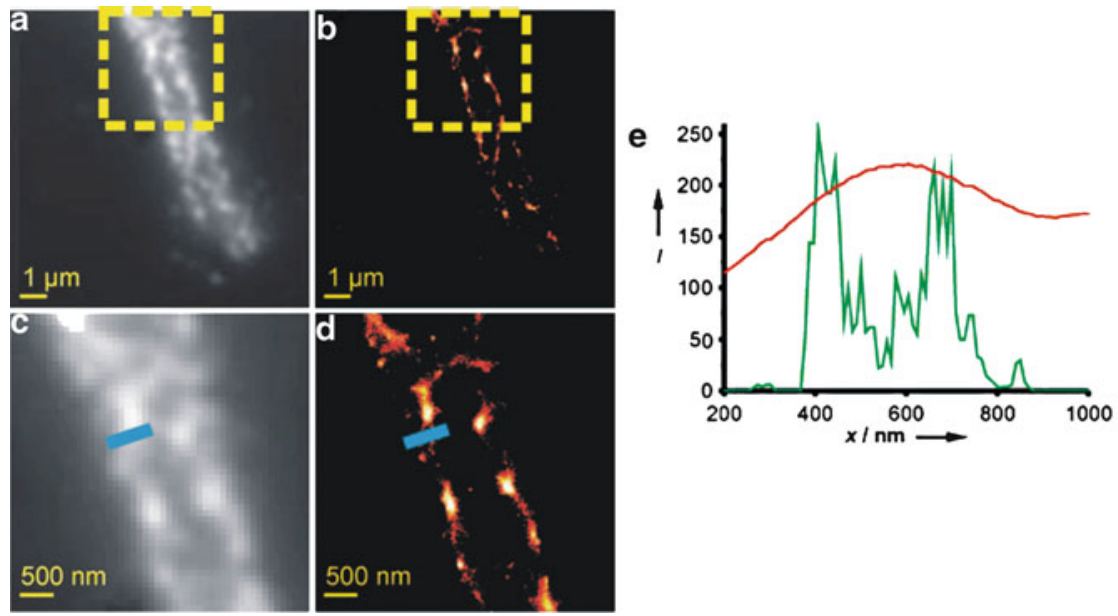

Fig. 8 Fluorescence microscopy-based imaging of labeled microtubules in fixed mouse fibroblasts. (a) Normal diffraction-limited fluorescence micrograph (accumulation of 8,000 frames). (b) Super-resolution image, reconstructed from localization of individual chromophore from the 8,000 frames in (a). (c, d) Zooming in on selected regions of, respectively, (a) and (b) clearly shows the difference in resolution. (e) Line profile showing the fluorescence intensity for the diffraction-limited (red) and the super-resolution image (green). Copyright Wiley-VCH Verlag GmbH \& Co. KGaA. Reproduced with permission from [23] 
an $\mathrm{ON}$ and an OFF state. Attaching such a chemical switch to a tubulin-antibody enables the visualization of microtubules within fibroblast cells. While many details are lost in the traditional diffraction-limited fluorescence image, localization of individual switching molecules enabled the authors to reconstruct a more detailed picture (Fig. 8). This approach has some advantages over PALM microscopy, since only one excitation source is required which is similar to GSDIM or dSTORM experiments [39, 40]. The authors indicate that the advantage over GSDIM or $\mathrm{dSTORM}$ is the independence of the ON-OFF switching on the excitation power. The fluorescent state of the probe can be controlled by the $\mathrm{Cu}$ (II) content in the cell.

While the use of $\mathrm{Cu}$ (II) ions in cells is not always desirable, a more general solution might come from probes from which the fluorescence emission is, for example, based on the binding of protons [20]. Loading chromophores with the right $\mathrm{p} K_{\mathrm{a}}$ would allow to probe biological structures with a NASCA microscopy imaging scheme.

\section{Conclusion and Perspectives}

This chapter summarizes the power of NASCA microscopy as a super-resolution optical technique. NASCA microscopy exploits the possibility of current fluorescence microscopes to observe individual chemical transformations of fluorogenic substrates. These conversions are recorded as diffraction-limited intensity profiles by a sensitive CCD camera. Mathematical fitting of this PSFs and this for many consecutive frames allows the reconstruction of high resolution images. Such NASCA micrographs reveal nanoscale features that would otherwise be lost in a diffraction-limited study. An obvious application of NASCA microscopy is the study of catalytic materials. Here not only high resolution activity mapping is possible; NASCA microscopy also allows quantification of local reactivity rates. Also in biological systems NASCA microscopy could reveal new information. Using fluorogenic substrates local enzymatic activity could be probed or structures of interest labeled with an enzyme could be targeted. Such an approach would be advantageous over other types of diffraction-unlimited optical microscopy since only one laser is required to visualize the products, and since bleaching of the attached chromophore does not occur, an enzyme-labeled structure can be targeted for many consecutive frames. On the other hand, probe molecules that switch between a fluorescent $\mathrm{ON}$ and OFF state when binding by analytes could also be envisioned. Chemical binding and unbinding of such biological substances like small molecules, proteins, etc. or even just protons could also give the necessary temporary signals necessary to allow a precise localization. Binding and unbinding events can also be directly monitored on very thin samples or on flat surfaces by using dye-bound reagents in combination with TIRF microscopy [32-35]. This eliminates the need for chromogenic reagents, and thus a wealth of different surface-related reactions can be assessed with this approach. 


\section{References}

1. Niemantsverdriet JW (2007) Spectroscopy in catalysis: An introduction. 3rd, Completely Revised and Enlarged Edition edn. Wiley-VCH, Weinheim

2. Weckhuysen BM (2009) Chemical imaging of spatial heterogeneities in catalytic solids at different length and time scales. Angew Chem Int Edit 48(27):4910-4943

3. Hulsken B, Van Hameren R, Gerritsen JW, Khoury T, Thordarson P, Crossley MJ, Rowan AE, Nolte RJM, Elemans J, Speller S (2007) Real-time single-molecule imaging of oxidation catalysis at a liquid-solid interface. Nat Nanotechnol 2(5):285-289. doi:10.1038/nnano.2007.106

4. de Smit E, Swart I, Creemer JF, Hoveling GH, Gilles MK, Tyliszczak T, Kooyman PJ, Zandbergen HW, Morin C, Weckhuysen BM, de Groot FMF (2008) Nanoscale chemical imaging of a working catalyst by scanning transmission x-ray microscopy. Nature 456 (7219):222-225

5. Urakawa A, Baiker A (2009) Space-resolved profiling relevant in heterogeneous catalysis. Top Catal 52(10):1312-1322. doi:10.1007/s11244-009-9312-3

6. Bergwerff JA, Lysova AA, Alonso LE, Koptyug IV, Weckhuysen BM (2007) Probing the transport of paramagnetic complexes inside catalyst bodies in a quantitative manner by magnetic resonance imaging. Angew Chem Int Edit 46:7224-7227. doi:10.1002/anie.200701399

7. Roeffaers MBJ, De Cremer G, Uji-i H, Muls B, Sels BF, Jacobs PA, De Schryver FC, De Vos DE, Hofkens J (2007) Single-molecule fluorescence spectroscopy in (bio)catalysis. P Natl Acad Sci USA 104(31):12603-12609

8. Roeffaers MBJ, Hofkens J, De Cremer G, De Schryver FC, Jacobs PA, De Vos DE, Sels BF (2007) Fluorescence microscopy: Bridging the phase gap in catalysis. Catal Today 126 (1-2):44-53

9. De Cremer G, Sels BF, De Vos DE, Hofkens J, Roeffaers MBJ (2010) Fluorescence micro (spectro)scopy as a tool to study catalytic materials in action. Chem Soc Rev 39(12):4703-4717

10. Hell SW (2009) Microscopy and its focal switch. Nat Methods 6(1):24-32. doi:10.1038/ nmeth.1291

11. Hell SW (2007) Far-field optical nanoscopy. Science 316(5828):1153-1158. doi:10.1126/ science. 1137395

12. De Cremer G, Roeffaers MBJ, Bartholomeeusen E, Lin K, Dedecker P, Pescarmona PP, Jacobs PA, De Vos DE, Hofkens J, Sels BF (2010) High-resolution single-turnover mapping reveals intraparticle diffusion limitation in ti-mcm- 41 catalyzed epoxidation. Angew Chem Int Edit 49(5):908-911

13. Roeffaers MBJ, De Cremer G, Libeert J, Ameloot R, Dedecker P, Bons A-J, Bückins M, Martens J, Sels BF, De Vos DE, Hofkens J (2009) Super-resolution reactivity mapping of nanostructured catalyst particles. Angew Chem Int Edit 48(49):9285-9289

14. Smiley RD, Hammes GG (2006) Single molecule studies of enzyme mechanisms. Chem Rev 106(8):3080-3094

15. Tachikawa T, Majima T (2010) Single-molecule, single-particle fluorescence imaging of tio2based photocatalytic reactions. Chem Soc Rev 39(12):4802-4819. doi:10.1039/b919698f

16. Chen P, Zhou XC, Shen H, Andoy NM, Choudhary E, Han KS, Liu GK, Meng WL (2010) Single-molecule fluorescence imaging of nanocatalytic processes. Chem Soc Rev 39 (12):4560-4570. doi:10.1039/b909052p

17. Roeffaers MBJ, Sels BF, Uji-i H, De Schryver FC, Jacobs PA, De Vos DE, Hofkens J (2006) Spatially resolved observation of crystal-face-dependent catalysis by single turnover counting. Nature 439(7076):572-575

18. Xu WL, Shen H, Kim YJ, Zhou XC, Liu GK, Park J, Chen P (2009) Single-molecule electrocatalysis by single-walled carbon nanotubes. Nano Lett 9(12):3968-3973. doi:10.1021/ n1900988f

19. Tachikawa T, Wang N, Yamashita S, Cui SC, Majima T (2010) Design of a highly sensitive fluorescent probe for interfacial electron transfer on a tio2 surface. Angew Chem Int Edit 49 (46):8593-8597. doi:10.1002/anie.201004976 
20. Ameloot R, Roeffaers MBJ, Baruah M, De Cremer G, Sels BF, De Vos DE, Hofkens J (2009) Towards direct monitoring of discrete events in a catalytic cycle at the single molecule level. Photochem Photobiol Sci 8(4):453-456

21. Kiel A, Jarve A, Kovacs J, Mokhir A, Kramer R, Herten DP (2007) Single-molecule studies on individual metal complexes. Proc of SPIE 6,444

22. Kiel A, Kovacs J, Mokhir A, Kramer R, Herten DP (2007) Direct monitoring of formation and dissociation of individual metal complexes by single-molecule fluorescence spectroscopy. Angew Chem Int Edit 46(18):3363-3366

23. Schwering M, Kiel A, Kurz A, Lymperopoulos K, Sprodefeld A, Kramer R, Herten DP (2011) Far-field nanoscopy with reversible chemical reactions. Angew Chem Int Edit 50 (13):2940-2945. doi:10.1002/anie.201006013

24. Roeffaers MBJ, Sels BF, Uji-i H, Blanpain B, L'Hoest P, Jacobs PA, De Schryver FC, Hofkens J, De Vos DE (2007) Space- and time-resolved visualization of acid catalysis in zsm-5 crystals by fluorescence microscopy. Angew Chem Int Edit 46(10):1706-1709

25. Roeffaers MBJ, Ameloot R, Bons A-J, Mortier W, De Cremer G, de Kloe R, Hofkens J, De Vos DE, Sels BF (2008) Relating pore structure to activity at the subcrystal level for zsm-5: An electron backscattering diffraction and fluorescence microscopy study. J Am Chem Soc 130(41):13516-13517

26. De Cremer G, Bartholomeeusen E, Pescarmona PP, Lin K, De Vos DE, Hofkens J, Roeffaers MBJ, Sels BF (2010) The influence of diffusion phenomena on catalysis: A study at the single particle level using fluorescence microscopy. Catal Today 157:236-242

27. Pap EHW, Drummen GPC, Winter VJ, Kooij TWA, Rijken P, Wirtz KWA, Op den Kamp JAF, Hage WJ, Post JA (1999) Ratio-fluorescence microscopy of lipid oxidation in living cells using c11-bodipy581/591. FEBS Lett 453(3):278-282

28. Roeffaers MBJ, Sels BF, De Schryver FC, Jacobs PA, Hofkens J, De Vos DE (2007) In situ filming of reactions inside individual zeolite crystals using fluorescence microscopy. Stud Surf Sci Catal 170:717-723

29. Kox MHF, Stavitski E, Weckhuysen BM (2007) Nonuniform catalytic behavior of zeolite crystals as revealed by in situ optical microspectroscopy. Angew Chem Int Edit 46 (20):3652-3655

30. Stavitski E, Kox MHF, Weckhuysen BM (2007) Revealing shape selectivity and catalytic activity trends within the pores of h-zsm- 5 crystals by time- and space-resolved optical and fluorescence microspectroscopy. Chem-Eur J 13(25):7057-7065

31. Kox MHF, Domke KF, Day JPR, Rago G, Stavitski E, Bonn M, Weckhuysen BM (2009) Labelfree chemical imaging of catalytic solids by coherent anti-stokes raman scattering and synchrotron-based infrared microscopy. Angew Chem Int Edit 48(47):8990-8994. doi:10.1002/ anie. 200904282

32. Canham SM, Bass JY, Navarro O, Lim SG, Das N, Blum SA (2008) Toward the single-molecule investigation of organometallic reaction mechanisms: single-molecule imaging of fluorophoretagged palladium(ii) complexes. Organometallics 27(10):2172-2175. doi:10.1021/om800228v

33. Lim SG, Blum SA (2009) A general fluorescence resonance energy transfer (fret) method for observation and quantification of organometallic complexes under reaction conditions. Organometallics 28(16):4643-4645. doi:10.1021/om900629s

34. Esfandiari NM, Wang Y, Bass JY, Cornell TP, Otte DAL, Cheng MH, Hemminger JC, McIntire TM, Mandelshtam VA, Blum SA (2010) Single-molecule imaging of platinum ligand exchange reaction reveals reactivity distribution. J Am Chem Soc 132(43):15167-15169. doi:10.1021/ja105517d

35. Esfandiari NM, Wang Y, McIntire TM, Blum SA (2011) Real-time imaging of platinum-sulfur ligand exchange reactions at the single-molecule level via a general chemical technique. Organometallics 30(11):2901-2907. doi:10.1021/om100911n

36. Sels BF, De Vos DE, Jacobs PA (2001) Hydrotalcite-like anionic clays in catalytic organic reactions. Catal Rev 43(4):443-488 
37. Tachikawa T, Yamashita S, Majima T (2011) Evidence for crystal-face-dependent tio2 photocatalysis from single-molecule imaging and kinetic analysis. J Am Chem Soc 133 (18):7197-7204. doi:10.1021/ja201415j

38. Wang N, Tachikawa T, Majima T (2011) Single-molecule, single-particle observation of sizedependent photocatalytic activity in au/tio2 nanocomposites. J Chem Sci 2(5):891-900. doi:10.1039/c0sc00648c

39. Heilemann M, van de Linde S, Schuttpelz M, Kasper R, Seefeldt B, Mukherjee A, Tinnefeld P, Sauer M (2008) Subdiffraction-resolution fluorescence imaging with conventional fluorescent probes. Angew Chem Int Edit 47(33):6172-6176. doi:10.1002/anie.200802376

40. Folling J, Bossi M, Bock H, Medda R, Wurm CA, Hein B, Jakobs S, Eggeling C, Hell SW (2008) Fluorescence nanoscopy by ground-state depletion and single-molecule return. Nat Methods 5(11):943-945. doi:10.1038/nmeth.1257 